\title{
Unexpectedly Large Couplings Between Orthogonal Units in Anthraquinone Polymers
}

\author{
Fornari, Rocco P.; de Silva, Piotr
}

Published in:

Chemistry - A European Journal

Link to article, DOI:

10.1002/chem.201903227

Publication date:

2019

Document Version

Peer reviewed version

Link back to DTU Orbit

Citation (APA):

Fornari, R. P., \& de Silva, P. (2019). Unexpectedly Large Couplings Between Orthogonal Units in Anthraquinone Polymers. Chemistry - A European Journal, 25(64), 14651-14658. https://doi.org/10.1002/chem.201903227

\section{General rights}

Copyright and moral rights for the publications made accessible in the public portal are retained by the authors and/or other copyright owners and it is a condition of accessing publications that users recognise and abide by the legal requirements associated with these rights.

- Users may download and print one copy of any publication from the public portal for the purpose of private study or research.

- You may not further distribute the material or use it for any profit-making activity or commercial gain

- You may freely distribute the URL identifying the publication in the public portal

If you believe that this document breaches copyright please contact us providing details, and we will remove access to the work immediately and investigate your claim. 


\section{CHEMISTRY A European Journal}

\section{Accepted Article}

Title: Unexpectedly Large Couplings Between Orthogonal Units in Anthraquinone Polymers

Authors: Rocco P. Fornari and Piotr de Silva

This manuscript has been accepted after peer review and appears as an Accepted Article online prior to editing, proofing, and formal publication of the final Version of Record (VoR). This work is currently citable by using the Digital Object Identifier (DOI) given below. The VoR will be published online in Early View as soon as possible and may be different to this Accepted Article as a result of editing. Readers should obtain the VoR from the journal website shown below when it is published to ensure accuracy of information. The authors are responsible for the content of this Accepted Article.

To be cited as: Chem. Eur. J. 10.1002/chem.201903227

Link to VoR: http://dx.doi.org/10.1002/chem.201903227 
WILEY-VCH

\section{Unexpectedly Large Couplings Between Orthogonal Units in Anthraquinone Polymers}

\author{
Rocco P. Fornari* and Piotr de Silva*
}

\begin{abstract}
We investigate the unusual electronic properties of directly linked 1,4-polyanthraquinones (14PAQ). The dihedral angle between the anthraquinones' (AQ) molecular planes is found to be close to $90^{\circ}$. Contrary to the prevailing notion that the interaction between orthogonal units is negligible due to the broken $\pi$-electron conjugation the couplings between neighboring $A Q$ units are found not to have a minimum at $90^{\circ}$ and to be much larger than expected. The unexpectedly large electronic coupling between orthogonal $A Q$ units is explained by the interaction between the lone pairs of the carbonylic oxygen and the $\pi$ system of the neighboring unit, which allows favorable overlap between frontier molecular orbitals at the orthogonal geometry. We show that this effect, which we describe computationally for the first time, can be strengthened by adding more quinone units. The effect of thermal fluctuations on the couplings is assessed through ab initio molecular dynamics simulations. The distributions of the couplings reveal that electron transport is resilient to dynamic disorder in all systems considered, while the hole couplings are much more sensitive to disorder. We describe lone pair$\pi$ interactions as a previously largely overlooked conjugation mechanism to be incorporated in a new class of disorder-resilient semiconducting redox polymers.
\end{abstract}

\section{Introduction}

Conjugated semiconducting polymers are an important class of materials in the field of organic electronics, where they have the potential to be a low-energy, low-cost and easily processable replacement for inorganic materials in some applications. ${ }^{[1-6]}$ They can conduct charges thanks to the properties of their electronic states which are determined by the interactions between frontier orbitals of the polymer's repeating units (monomers). These $\pi$ orbitals allow the excess charges to delocalize over the polymer backbone. The latter property is essential for efficient charge transport along the polymer chain, which is one of the main factors determining bulk mobility. ${ }^{[7-9]}$ Due to the nature of $\pi$ orbitals, the conjugation across monomers is optimal when they are coplanar. ${ }^{[1,3,10]}$ The charge transfer integral (electronic coupling) between neighboring monomers depends on the inter-monomer torsion (dihedral angle) and decreases when the geometry deviates from planarity. ${ }^{[10-13]}$ It appears obvious that one of the most successful design strategies for improving the mobility of semiconducting polymers has been maximizing intra-chain conjugation by synthetizing coplanar monomers and

Dr. R. P. Fornari, Dr. P. de Silva

Department of Energy Conversion and Storage

Technical University of Denmark

Fysikvej 309, 2800 Kongens Lyngby, Denmark

E-mail: rocfor@dtu.dk,pdes@dtu.dk

Supporting information for this article is given via a link at the end of the document. minimizing the freedom of rotation..$^{[1,6,10]}$ The torsion between aromatic rings can be hindered for example by an $\mathrm{sp}^{3}$ carbon connecting them, leading to almost planar monomers such as fluorene. The $\sim 100$ times higher mobility of Poly(9,9-dioctylfluorene) (PFO) compared to Poly(p-phenylene vinylene) (PPV) derivatives was attributed to the hindered torsion between aromatic rings leading to larger inter-monomer couplings and more delocalized charge carriers. ${ }^{[14]}$ More recently, monomers planarized in a similar fashion have been incorporated in some of the best performing donor-acceptor polymers such as CDT-BTZ ${ }^{[15,16]}$ and IDT-BT. ${ }^{[17,18]}$ Another successful strategy is removing the hydrogens adjacent to the bond between aromatic rings (which sterically prevent coplanarity of the monomers) by substituting the $\mathrm{C}-\mathrm{H}$ groups with nitrogen and sulphur, obtaining a perfectly planar monomer whose polymer has high electron mobility. ${ }^{[19]}$ Coplanarity can also be promoted by inter-monomer weak bonding between carbonyl oxygens and $\mathrm{S}$ or $\mathrm{H}^{\left[{ }^{[10]}\right.}$ The inevitable conformational disorder of a polymer chain means there is a distribution of dihedral angles generating static disorder in site energies and couplings which causes localization of the electronic states. ${ }^{[20]}$ Transport will also be affected by dynamic disorder due to nuclear motions on time scales comparable or faster than that of charge transport. ${ }^{[21-23]}$

Theoretical and computational studies play a fundamental role in understanding and predicting structures and properties of semiconducting polymers. The bulk structure of polymers can be predicted by molecular dynamics (MD) simulations. The electronic states of the thereby generated conformations can be computed with quantum-chemical methods (MD/QC schemes) ${ }^{[20,24-28]}$ and charge transport can be described with various theoretical models. ${ }^{[29-35]}$ Using a combination of $M D$, quantum chemistry and transport models the dependence of charge transport properties on structure, temperature, field and charge density can be predicted. ${ }^{[36-39]}$ Multiscale simulation schemes combining models spanning different length and time scales, ${ }^{[25,40-45]}$ which often involve some form of coarse graining help to reveal the impact of local and bulk-scale structure on charge transport. ${ }^{[7]}$ While the development of such comprehensive models is certainly beneficial to a more general understanding of polymeric materials, simple electronic structure calculations on dimer geometries along the dihedral angle coordinate can already provide valuable insights into atomic scale structure-property relationships. ${ }^{[11,12,46]}$

Troisi and Shaw have recently shown that some dimers have a surprising property: the coupling between monomers is almost insensitive to rotations of the dihedral angle up to $\sim 60^{\circ}$ from planarity. ${ }^{[4]}$ This unusual conjugation at large dihedral angles was ascribed to a favorable overlap between the HOMOs of the monomers. Here we discuss a different but related phenomenon that appears to contradict the current understanding of conjugated polymers. We show that the electronic coupling between directly linked anthraquinones is surprisingly large despite their molecular planes being nearly orthogonal at the equilibrium geometry. The synthesis and characterization of 
directly linked polyanthraquinones (PAQ) was first reported by Yamamoto and coworkers. ${ }^{[48-50]}$ Only in recent years PAQ (both directly and indirectly linked) have been shown to be viable candidates as electroactive cathode materials for $\mathrm{Li}^{-i o n^{[51,52]} \text { and }}$ $\mathrm{Mg}$-ion ${ }^{[53,54]}$ batteries. In these reports, the 1,4 directly linked $P A Q$ (14PAQ) was found to have excellent electrochemical properties (fast and reversible redox behavior, high capacity retention and cycling stability). However, its charge transport properties, which also impact the overall electrochemical performance, were not discussed in refs. ${ }^{[51,54]}$. The conductivity of 14PAQ and other directly linked polyanthraquinones was reported to be appreciable in the undoped state. ${ }^{[50]}$ In order to explain the apparent contradiction between the orthogonal geometry, which in principle should break the conjugation, and the experimental evidence of good electronic coupling, we compute, analyze and discuss the geometry, orbitals and charge transfer integrals of 14PAQ and the double quinone 1,4-polyanthracenetetrone (14PAT) and propose a new molecular design strategy based on these observations.

\section{Results and Discussion}

The electronic properties of a polymer chain are determined mainly by the interactions between neighboring monomers. Therefore, a great deal can already be learned by analyzing and discussing the properties of the 1,4-linked $A Q$ dimer. Bulk properties depend on chain conformation, packing, solvent (if present) and interchain interactions which are all aspects outside the scope of this paper. Both electrochemical and charge transport properties are largely determined by the frontier orbitals (HOMO and LUMO) of the monomers. The motivation for studying $14 \mathrm{PAQ}$ stems from its promising performance as organic cathode material in batteries. The goal of this paper is however a more general understanding of the properties of this class of polymeric quinones. Since in principle both electron and hole transport can be operational at the same time in organic semiconductors, ${ }^{[55,56]}$ we are interested in the possible states of both quasiparticles and we begin the discussion with the electron couplings.

At the equilibrium geometry the dihedral angle between the $A Q$ units is almost orthogonal $\left(91.6^{\circ}\right)$, probably mainly due to steric repulsion ${ }^{[46]}$ between the hydrogens in positions 2 and 3 and the carbonyl oxygens, but also attractive interactions may play a role in determining this geometry and will be discussed later. The energies and shapes of the frontier orbitals of $A Q$ and of its dimer are shown in Figure $\mathrm{S} 1$ (Supporting Information). The LUMO and LUMO +1 of the dimer can be clearly interpreted as linear combinations of the LUMOs of the monomers, which have the typical aromatic hydroquinonic shape. The nodal structure of these LUMOs allows for favorable overlap between the oxygen's lone pair and the aromatic system of the neighboring monomer at the nearly orthogonal geometry. A closer inspection of the dimer's LUMO (Figure 1) reveals that the oxygen's lone pair interacts with both the quinone unit and the aromatic (backbone) ring. Experimental evidence of such carbonyl- $\pi$ interactions in the 1,4 linked $A Q$ dimer and polymer was reported by Gautrot et al. ${ }^{[57]}$ The $0.08 \mathrm{eV}$ energy splitting between LUMO and LUMO+1 points to a non-negligible LUMO-LUMO coupling of about $0.04 \mathrm{eV}$, which is surprisingly large given the nearly orthogonal geometry. In order to explain this unusual observation, we compute the electron transfer integrals as a function of the dihedral angle between the $A Q$ units with the two methods described in the previous section: i) fragment orbital (FO), i.e. coupling between the monomers' MO (e.g. J JuMO, J JOMO) and ii) CDFT-Cl coupling

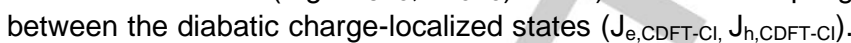
The former is often assumed to be a reasonable choice for the theoretical description of charge transport in polymers, but it is valid only when the monomer's transport-relevant $\mathrm{MO}$ can be considered energetically well-separated from the other MO so that there is negligible mixing between different MOs. Furthermore, since FO couplings are computed for neutral fragments, they neglect the polarization and orbital relaxation effects due to the charge on the other unit, which are instead captured by the CDFT-CI method. ${ }^{[58,59]}$

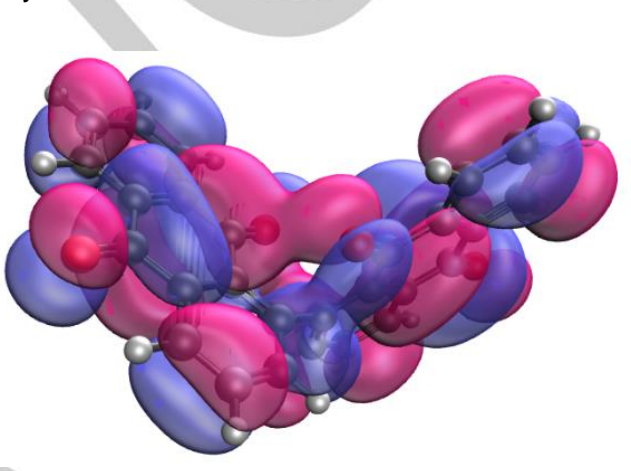

Figure 1. LUMO of the $14 \mathrm{AQ}$ dimer (iso value 0.02).

As shown in the top panel of Figure 2, both methods predict that the electron coupling between $A Q$ units does not have a minimum around $90^{\circ}$, unlike conventional conjugated polymers. ${ }^{[13,47]}$ The minima of $\left|\mathrm{J}_{\text {LUMO }}\right|$ and $\left|\mathrm{J}_{\text {e,CDFT-Cl }}\right|$ occur instead at $120^{\circ}$ and $140^{\circ}$ respectively. It is worth noting that, although their angle dependences are qualitatively similar, there is a significant deviation between the two coupling calculation methods: |J LUMO| significantly underestimates the CDFT-Cl coupling by almost 0.1 $\mathrm{eV}$ at the $90^{\circ}$ geometry. To distinguish the various components of the coupling, we examine a model system where the geometries are the same as for the $A Q$ dimer, but the atoms belonging only to the poly-paraphenylene-like backbone have been removed. The remaining naphthoquinone (NQ) fragments (structure shown in the bottom panel of Figure 2) have been capped with hydrogens. In this backboneless model system, there is almost a complete agreement between the two methods, suggesting that the polarization effect is almost negligible for the coupling between nonbonded NQ fragments. The polarization effects missed by the FO method are much stronger in the $A Q$ dimer, and there are two reasons for this: i) the partially negatively charged oxygen interacts favorably not only with the quinone unit but also with the electron-deficient aromatic backbone ring ${ }^{[57]}$ and this effect is much stronger when the extra electron is explicitly included in the calculation (CDFT-Cl method); ii) the polarization effects are stronger for chemically bonded units due to inductive effect (through-bond polarization). The qualitatively similar behavior of the CDFT-Cl couplings in the AQ dimer and in the backboneless model system confirms that the coupling is mainly determined by 
the interaction between the LUMOs, although the CDFT-CI method is certainly the preferable choice for computing accurate couplings between bonded units.
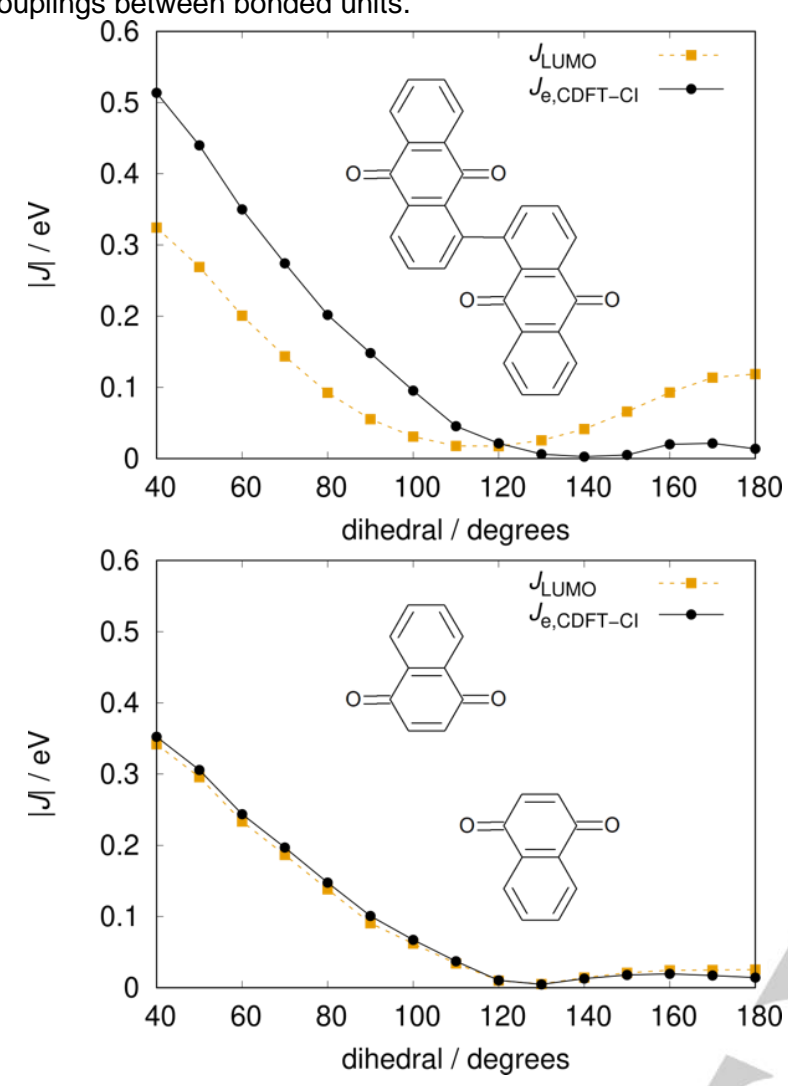

Figure 2. Electron transfer integral as a function of the dihedral angle between anthraquinone units (top) and after removal of the backbone (bottom). The two different methods indicated in the legend are described in the text.

We now move on to the discussion of hole couplings. The HOMO and $\mathrm{HOMO}-1$ of the dimer seem to originate from a mixing between HOMO and HOMO-1 of the monomers (see Figure S1, Supporting Information), where the former has a partial $n$ character (large density on the oxygen's lone pairs) and the latter is aromatic ( $\pi$ character). The resulting HOMO and HOMO- 1 of the dimer are destabilized with respect to the monomer and the $\pi-\Pi^{*}$ optical transition is therefore red-shifted as observed by Gautrot et al. ${ }^{[57]}$, who had instead attributed the red-shift to the stabilization of the LUMO. Here the FO method is useful to interpret the results since it is possible to compute the couplings between pairs of different orbitals to assess the magnitude of their interaction at different geometries. The dihedral angle dependences of the three FO couplings between HOMO and HOMO-1, shown in Figure 3, are very different from each other. $\left|\mathrm{J}_{\mathrm{HOMO}}\right|$ has a minimum at $110^{\circ}$ and does not vary in a wide range, i.e. the interaction between the HOMOs (which have $\mathrm{n}$ character) is not very sensitive to the dihedral rotation; $\left|J_{\text {HOMO-1 }}\right|$ has an angle dependence more typical of aromatic units: it is very small at $90^{\circ}$ and grows sharply when moving towards more flat geometries; the coupling between $\mathrm{HOMO}$ and $\mathrm{HOMO}-1$ on different monomers, |J $\mathrm{J}_{\text {HOMO-HOMO-1 }} \mid$, is rather large at most geometries and has a maximum at $90^{\circ}$. It is clear that in this case none of the FO couplings is a reliable approximation of the transfer integral, contrary to the common assumption that the HOMO-HOMO coupling is a good indication of the hole transporting ability. The CDFT-Cl coupling $\left|\mathrm{J}_{\mathrm{e}, \mathrm{CDFT}-\mathrm{Cl}}\right|$, which captures the global effect by spatially constraining the charge on each of the two monomers but not on specific orbitals, is a more reliable measure of the charge transfer integral in this case. Its angular dependence has a minimum of $0.06 \mathrm{eV}$ at $90^{\circ}$ but it quickly grows when the angle is rotated in either direction: already at the $91.6^{\circ}$ equilibrium geometry $\left|\mathrm{J}_{\mathrm{e}, \mathrm{CDFT}-\mathrm{Cl}}\right|=0.15 \mathrm{eV}$.

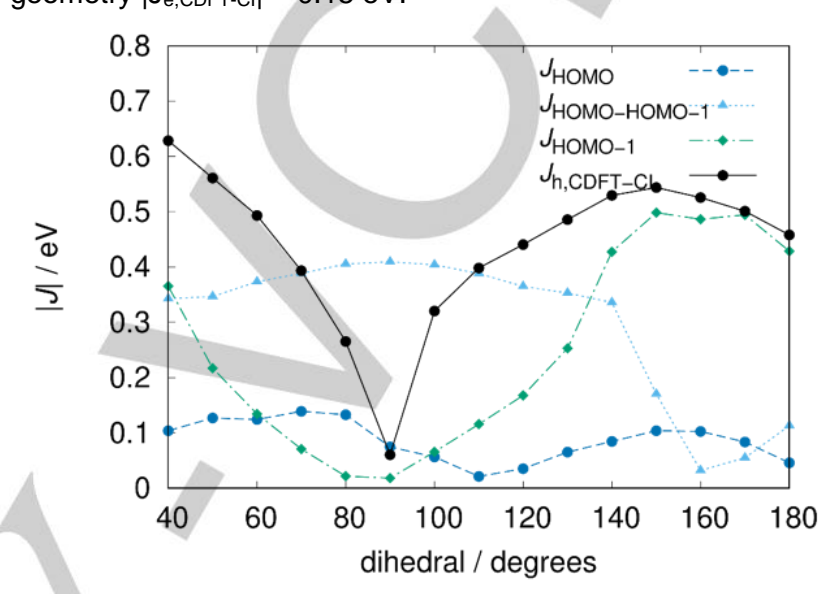

Figure 3. Hole transfer integral as a function of the dihedral angle between anthraquinone units. The two different methods indicated in the captions are described in the text.

The angular dependence of the three FO couplings between HOMO and HOMO-1 (Figure 3) can be better understood by looking at the orbital diagrams in Figure 4 . At $90^{\circ}$ both $\left|\mathrm{J}_{\text {HOMO }}\right|$ and $\left|\mathrm{J}_{\text {HOMO-1 }}\right|$ are rather small, but the strong $\left|\mathrm{J}_{\text {НОМО-Номо-1 }}\right|$ interaction causes a large $\sim 1 \mathrm{eV}$ splitting between the resulting orbitals in the dimer. These dimer orbitals (two bonding and two antibonding, pairwise degenerate) clearly originate from the mixing between HOMO and HOMO- 1 of the two monomers. This strong $n-\pi$ interaction is enabled by the small energy gap between them but especially because of their large mutual overlap. Simultaneous satisfaction of these two requirements is a prerequisite for efficient coupling and its realization at the near-orthogonal equilibrium geometry makes 14PAQ a rather unusual system. The lowest bonding orbital resulting from the HOMO-HOMO-1 mixing, shown in the top right panel of Figure 4, clearly illustrates the bonding interaction between one of the oxygen's lone pairs and the $\pi$ system of the other monomer. When the angle is constrained to $180^{\circ}$ (Figure 4 , bottom) the repulsion between the oxygen and the hydrogen of the other monomer causes a distortion from planarity. This may explain why the HOMO-1 of the monomer is slightly destabilized and acquires partial $\mathrm{n}$ character. At this geometry both the $\left|\mathrm{J}_{\text {Hомо-номо-1 }}\right|$ and $\left|\mathrm{J}_{\text {HOMO }}\right|$ couplings are weak as the overlap between the oxygens' lone pairs and the $\pi$ systems is negligible. The planarity of the aromatic backbone favors instead the $\left|\mathrm{J}_{\text {номо-1 }}\right|$ interaction. The two resulting dimer orbitals are split by almost $1.7 \mathrm{eV}$ and have a clear aromatic character. The resulting bonding orbital is shown in the bottom right panel of Figure 4. 


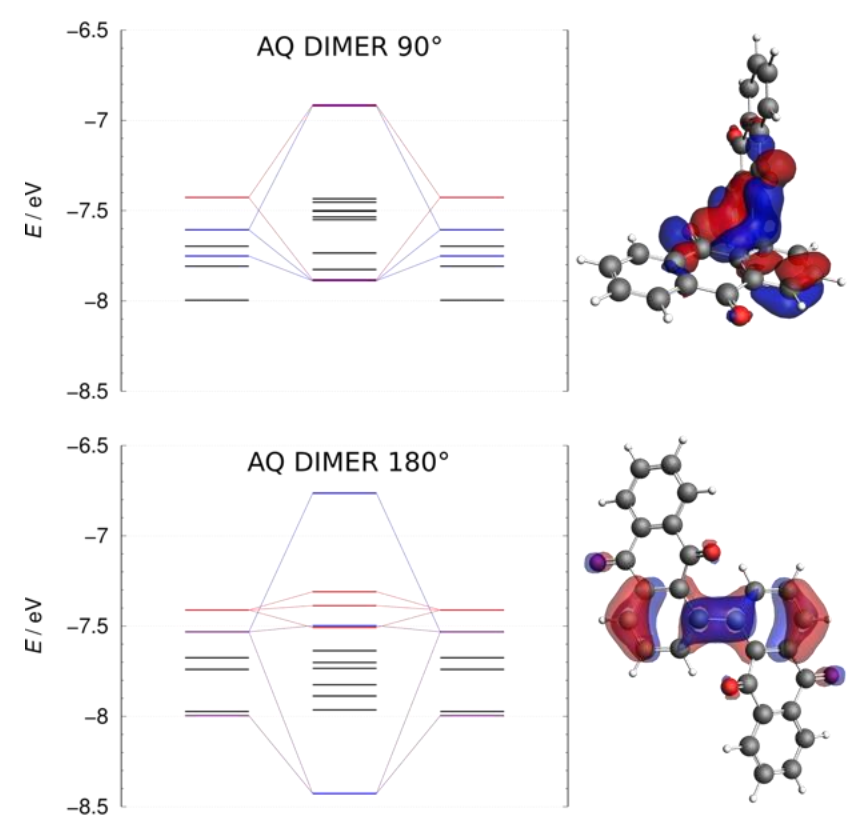

Figure 4. Orbital diagrams of the HOMOs of the monomer fragments and of the dimer at the $90^{\circ}$ and $180^{\circ}$ constrained geometries. Orbitals with predominantly $\mathrm{n}$ character (high density on the oxygen lone pairs) are represented in red; orbitals with predominantly $\pi$ character (aromatic) are represented in blue; orbitals with mixed $n-\pi$ character are represented in purple. The shape of the lowest dimer orbital in each diagram (with bonding character) is shown on the right (iso value 0.03 ). angles found in the AIMD snapshots, shown in the top panel of Figure 5, is found to be approximately Gaussian and the mean value $91.6 \pm 13.1^{\circ}$ is identical to the equilibrium geometry. The distribution of $\left|\mathrm{J}_{\mathrm{e}, \mathrm{CDFT}-\mathrm{Cl}}\right|$ is also approximately Gaussian and the average coupling $0.13 \pm 0.08 \mathrm{eV}$ (Boltzmann average $0.13 \mathrm{eV}$ ) is comparable to the equilibrium value $0.15 \mathrm{eV}$. The large difference between the distributions of $\left|\mathrm{J}_{\mathrm{e}, \mathrm{CDFT}-\mathrm{Cl}}\right|$ and $\left|\mathrm{J}_{\text {LUMO }}\right|$ confirms that the CDFT-Cl method is preferable for computing accurate couplings. The distribution of the hole transfer coupling |J $\mathrm{J}_{\mathrm{h}, \mathrm{CDFT}-\mathrm{Cl} \mid}$ is instead much broader and appears to be multimodal, with many points below $0.1 \mathrm{eV}$. The average $0.29 \pm 0.17 \mathrm{eV}$ (Boltzmann average $0.34 \mathrm{eV}$ ) is remarkably higher than the value obtained at the equilibrium geometry $(0.15 \mathrm{eV})$. This confirms that the independence of electronic couplings from nuclear coordinates (Condon approximation) is not valid in this case as was previously noted for the coupling between phenyl rings. ${ }^{[12]}$ The impact of thermal disorder on charge transport is difficult to predict without calculations on a larger scale, ${ }^{[25,27]}$ and the thermally averaged coupling may lead to an artificial overestimation of mobility. [61] Should the high sensitivity to nuclear displacements determine a similar distribution of couplings along a disordered polymer chain (i.e. a similar amount of static disorder), the subsequent localization of the transport states would be expected to negatively impact hole transport with respect to electron transport. ${ }^{[34]}$
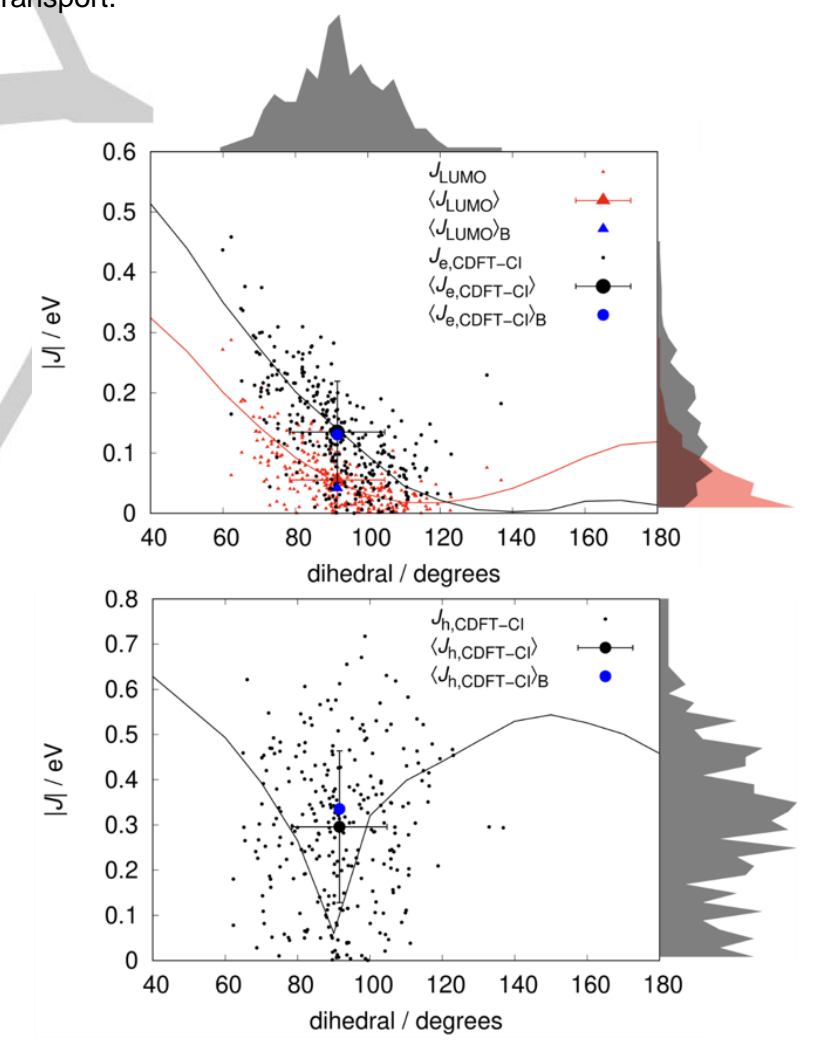

Figure 5. Electron (top) and hole (bottom) transfer integrals as a function of the dihedral angle: comparison between the relaxed scan (solid lines, same data as in Figure 2 and Figure 3), and 300 AIMD snapshots (small symbols). The average and standard deviation of the AIMD data is represented by larger symbols with error bars. Blue symbols represent the Boltzmann-weighted average $\sum|J| e^{-E / k_{\mathrm{B}} \mathrm{T}} / \sum e^{-E / k_{\mathrm{B}} \mathrm{T}}$. The distributions of the angles and couplings from AIMD are shown in the side panels. 
The magnitude and distribution of the computed charge transfer integrals is consistent with the experimental observation of a non-negligible conductivity of 14PAQ in the undoped state (10-8 $\mathrm{S} \mathrm{cm}-1)^{[50]}$ which is comparable to the undoped conductivity of redox polymers specifically designed to be conducting such as $\mathrm{P}(\mathrm{NDI} 2 \mathrm{OD}-\mathrm{T2}){ }^{[62]}$ In the latter example, the conductivity increases by 5 orders of magnitude upon n-doping. Given the excellent electron-accepting ability of $14 \mathrm{PAQ}$, it is reasonable to suggest that also its conductivity would increase dramatically with the high level of $n$-doping it would be subject to during operation as an active cathode material.

The favorable overlap between the oxygen's lone pairs and the aromatic system of the neighboring monomer is responsible for the large couplings for both electron and hole transfer. The main feature enabling this strong lone pair- $\pi$ inter-monomer interaction is the presence of carbonyl bonds parallel to the polymer backbone. It is likely that introducing more quinone units would strengthen this interaction. Therefore, to verify if this can be a successful molecular design strategy, we analyze next the properties of 1,4-polyanthracenetetrone (14PAT) and its partially reduced and protonated form 5,8-dihydroxy-1,4-polyanthraquinone (14PDHAQ) which was found to have a conductivity $\sim 100$ times larger than 14PAQ. ${ }^{[50]}$

The frontier orbital energies, equilibrium geometries and relevant orbital shapes of AT and DHAQ monomers and corresponding dimers are shown in Figure S2 (Supporting Information). At the equilibrium geometry of the 14PAT dimer the monomers' planes are bent, with the quinone units tending to stack on each other and a dihedral angle of $59.4^{\circ}$ between the aromatic rings. The equilibrium geometry of the 14PDHAQ dimer is instead more similar to 14PAQ with planar monomers and an identical dihedral angle of $91.6^{\circ}$. This difference can be due to the fact that both in 14PAQ and 14PDHAQ two of the three rings are aromatic (therefore favoring a more planar geometry) while in 14PAT only the linked ring retains aromaticity. The shapes of the LUMO and LUMO+2 of the 14PAT dimer illustrate how the attractive interaction between the quinone units is enabled by favorable overlap between LUMO and LUMO+1 of the monomers respectively. The HOMO of the 14PAT dimer results from a mixing between the monomers' $n$ and $\pi$ orbitals in a fashion similar to 14PAQ. In the 14PDHAQ dimer the HOMO is just formed by the hydroquinonic HOMOs (which are essentially AT's LUMOs occupied by two electrons). Their interaction is weak due to small mutual overlap at the orthogonal geometry, maybe because the electron density is larger on the reduced quinone (the one further away from the backbone) leading to smaller lone pair- $\pi$ interaction. The LUMO of the 14PDHAQ dimer is almost identical to the LUMO of $14 \mathrm{PAQ}$ and a similar interaction mechanism is expected.

Figure 6 summarizes the dependence of the CDFT-Cl couplings in 14PAT and 14PDHAQ on the dihedral angle along the relaxed scan coordinate and from 300 AIMD snapshots. The AIMD simulations were run at $\mathrm{T}=300 \mathrm{~K}$, the total simulation time was $38.7 \mathrm{ps}$ and after $9.7 \mathrm{ps}$ equilibration time a snapshot was taken every 100 time steps. The distribution of dihedral angles from the AIMD is approximately Gaussian in both dimers, but in 14PAT the average angle $89 \pm 14^{\circ}$ is much larger than the equilibrium angle $59.4^{\circ}$ while in $14 \mathrm{PDHAQ}$ the average angle $90 \pm 12^{\circ}$ is closer to the equilibrium value $91.6^{\circ}$. A possible explanation for this difference is that the attractive interaction between the double quinones in 14PAT causes the global minimum to be at a smaller angle, which is however easily escaped at $300 \mathrm{~K}$ : the potential energy surface along the scan coordinate was found to be very shallow and is consistent with the range of angles $\left(50-120^{\circ}\right)$ explored by the AIMD (see Figure 7).

In 14PAT (Figure 6, top) the electron transfer integral $\left|\mathrm{J}_{\mathrm{e}, \mathrm{CDFT}-\mathrm{Cl} \mid}\right|$ is remarkably large along the relaxed scan: at the equilibrium geometry it is as high as $0.27 \mathrm{eV}$ and at the $80^{\circ}$ constrained geometry it reaches a rather large minimum value of $0.20 \mathrm{eV}$. The average $\left|\mathrm{J}_{\mathrm{e}, \mathrm{CDFT}-\mathrm{Cl}}\right|$ from the AIMD snapshots is also large $(0.21 \pm$ $0.06 \mathrm{eV}$, Boltzmann average $0.20 \mathrm{eV}$ ) and it has a rather narrow distribution which suggests it is resilient to dynamic disorder leading to efficient electron transport (assuming that the distribution of couplings due to static disorder is not much wider). The $\left|J_{\text {e,CDFT-Cl }}\right|$ data also show a correlation with the dihedral angle, but it is even weaker than in 14PAQ (see Figure 5). The hole transfer integral $\left|\mathrm{J}_{\mathrm{h}, \mathrm{CDFT}-\mathrm{Cl}}\right|$ is comparable to $\left|\mathrm{J}_{\mathrm{e}, \mathrm{CDFT}-\mathrm{Cl}}\right|$ along the relaxed scan but the AIMD snapshots show much lower couplings: the distribution of $J_{\mathrm{h}, \mathrm{CDFT}-\mathrm{Cl}}$ is centered around 0 and the average of $\left|\mathrm{J}_{\mathrm{h}, \mathrm{CDFT}-\mathrm{Cl}}\right|$ is $0.07 \pm 0.05 \mathrm{eV}$. As in $14 \mathrm{PAQ}$, the hole transfer integrals from the AIMD show no correlation at all with the dihedral angle. The relative magnitude and distributions of $\left|\mathrm{J}_{\mathrm{e}, \mathrm{CDFT}-\mathrm{Cl}}\right|$ and $\left|\mathrm{J}_{\mathrm{h}, \mathrm{CDFT}-\mathrm{Cl}}\right|$ suggest that electron transport should be more efficient than hole transport in 14PAT.

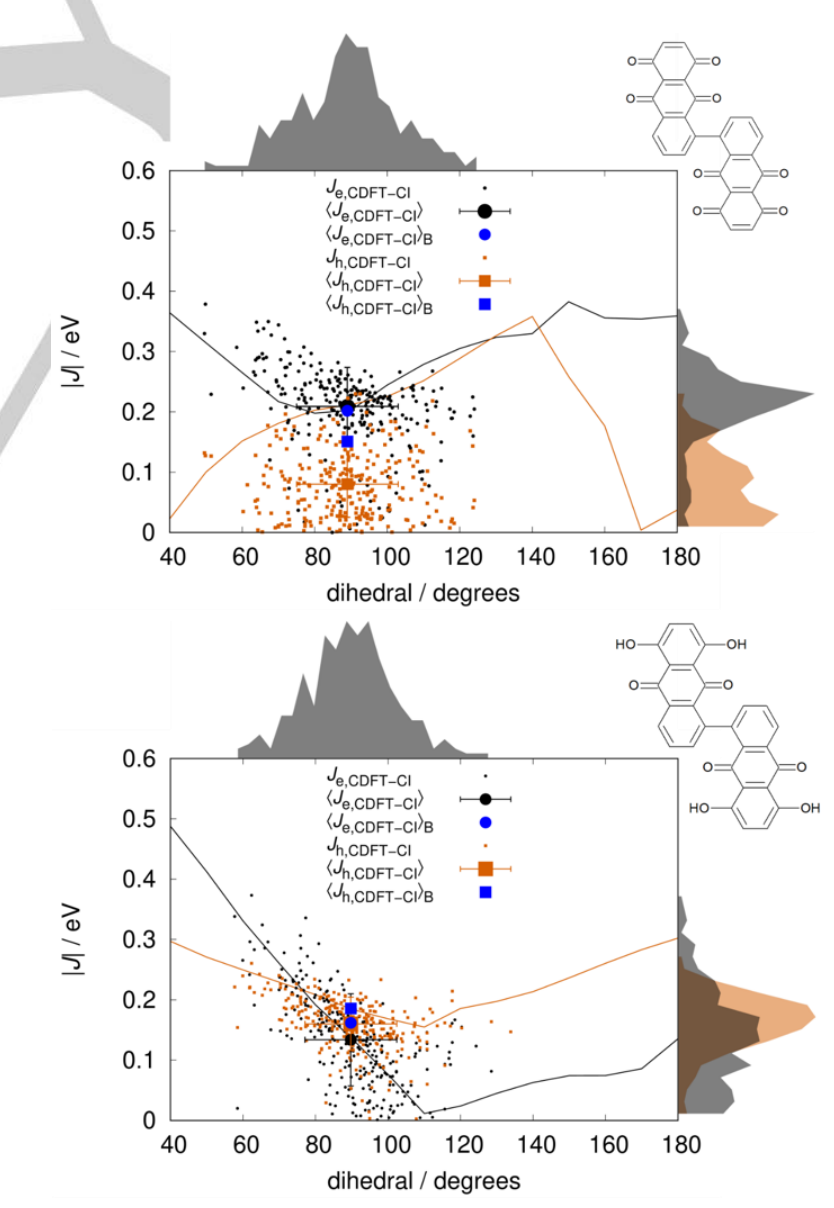

Figure 6. Electron and hole transfer integrals in 14PAT (top) and 14PDHAQ (bottom) as a function of the dihedral angle: comparison between the relaxed 
scan (lines) and 300 AIMD snapshots (small points). The average and standard deviation of the AIMD data is represented by larger symbols with error bars Blue symbols represent the Boltzmann-weighted average $\sum|J| e^{-E / k_{\mathrm{B}} \mathrm{T}} / \sum e^{-E / k_{\mathrm{B}} \mathrm{T}}$ The distributions of the angles and couplings from AIMD are shown in the side panels.

In 14PDHAQ (Figure 6, bottom) the angle dependence of $\left|\mathrm{Je}_{\mathrm{e}, \mathrm{CDFT} \text {-Cl }}\right|$ is similar to that of $14 \mathrm{PAQ}$ since their LUMOs are electronically equivalent and the equilibrium value is $0.135 \mathrm{eV}$. The distribution of $\left|\mathrm{J}_{\mathrm{e}, \mathrm{CDFT}-\mathrm{Cl}}\right|$ obtained from the AIMD snapshots is wide and flat with average $0.13 \pm 0.08 \mathrm{eV}$ (Boltzmann average $0.16 \mathrm{eV}$ ) and the data, widely scattered around the relaxed scan trend, shows moderate correlation with the dihedral angle. The angle dependence of the hole transfer integral $\left|\mathrm{J}_{\mathrm{h}, \mathrm{CDFT}-\mathrm{Cl}}\right|$ along the relaxed scan coordinate is qualitatively similar to $\left|\mathrm{Je}_{\mathrm{e}, \mathrm{CDFT}-\mathrm{Cl} \mid}\right|$ of 14PAT: the equilibrium value is $0.19 \mathrm{eV}$ and it reaches a minimum of $0.15 \mathrm{eV}$ at $110^{\circ}$. The distribution of $\left|\mathrm{J}_{\mathrm{h}, \mathrm{CDFT}-\mathrm{Cl} \mid}\right|$ is also similar to that of $\left|\mathrm{J}_{\mathrm{e}, \mathrm{CDFT}-\mathrm{Cl}}\right|$ in 14PAT and shows a weak correlation with the dihedral angle. Its average value is $0.16 \pm 0.04 \mathrm{eV}$ (Boltzmann average $0.19 \mathrm{eV}$ ). These results suggest that both holes and electrons can be efficiently transported in 14PDHAQ and may explain the higher conductivity compared with 14PAQ measured by Yamamoto et al. ${ }^{[50]}$.

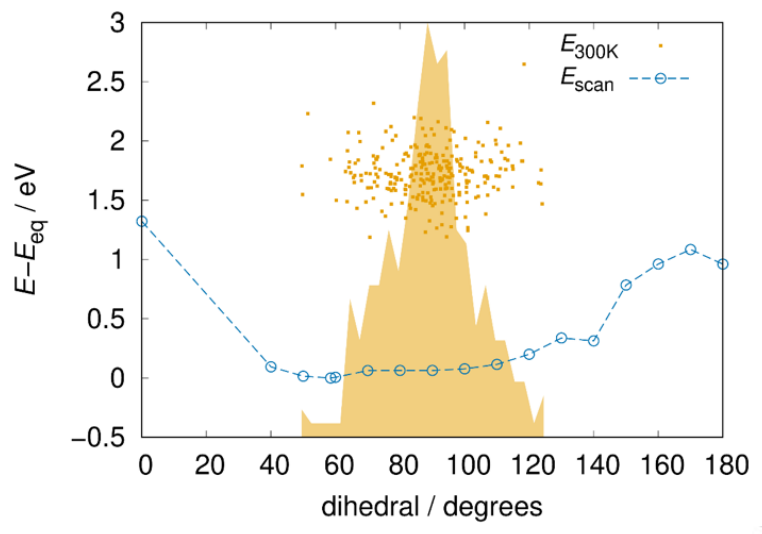

Figure 7. Energies of the relaxed scan structures $\left(B 3 L Y P / 3-21 G^{*}\right)$ along the dihedral angle coordinate, electronic potential energies of the 14PAT dimer in the 300 AIMD snapshots and distribution of their dihedral angles. The energy of the equilibrium structure $E_{\mathrm{eq}}$ is taken as 0 .

\section{Conclusion}

We have found unexpectedly large hole and electron transfer integrals between directly 14 -linked $A Q$ units despite the nearly orthogonal geometry. The main feature responsible for this counterintuitive property was found to be the interaction between the lone pairs on the oxygen of the carbonyl groups and the aromatic system of the neighboring monomer. Both occupied and unoccupied frontier orbitals were found to play a role in this interaction. The favorable overlap at orthogonal geometry between the monomers' hydroquinonic LUMOs is shown to be the main reason behind the high electron transfer integrals observed. In the case of the occupied orbitals, a strong mixing between the $n$-type HOMO and the $\pi$-type HOMO- 1 was found to be responsible for the observed large hole transfer integral. In both cases the oxygen's lone pairs interact with both the aromatic backbone ring and the quinone unit at nearly orthogonal geometries. The narrow distribution of electron transfer integrals obtained from AIMD at $300 \mathrm{~K}$ suggests that in 14PAQ electron transport might be rather resilient to dynamic disorder while hole transfer integrals are much more sensitive to nuclear displacements. The effect of doubling the number of quinone units was assessed by analyzing 14PAT and its reduced and protonated form 14PDHAQ. The higher electron transfer couplings obtained for 14PAT, combined with their high resilience to dynamic disorder, confirm that adding more carbonyl units is a viable design strategy to improve electron transport. 14PDHAQ exhibits a good balance between hole and electron transfer couplings which suggests that ambipolar charge transport may be responsible for the higher experimental conductivity compared to 14PAT.

In summary, the interaction between the carbonylic oxygen's lone pairs and the aromatic system of neighboring monomers can lead to an efficient charge transport despite large dihedral angles between the monomers. This property has been largely overlooked both in the basic physical organic chemistry as well as organic electronics literature. The detailed insights provided in this contribution can help in designing new high-performance, disorder-tolerant semiconducting and redox-active polymers incorporating quinone units with carbonyl bonds parallel to the polymer backbone, which could find applications both in the field of energy storage and in polymer electronics.

\section{Computational Section}

All geometry optimizations are carried out in vacuum at the B3LYP/6-31G** level of theory using the Q-Chem 5.0 software package. [63] The geometries for the dihedral angle scan (referred to as relaxed scan) are obtained by constraining the angle between the linked aromatic rings to the desired value while optimizing all the other degrees of freedom.

The electron and hole transfer integrals are computed with the constrained density functional theory configuration interaction (CDFT-Cl) method developed by Wu and Van Voorhis ${ }^{[64,65]}$ as implemented in Q-Chem 5.0. The CDFT-Cl coupling is the interaction between two charge-localized diabatic states, each obtained by constraining one net charge on one of the fragments forming the dimer.

The fragment orbital (FO) couplings are computed at the B3LYP/AUG-ADZP level of theory using the ADF 2017 software. ${ }^{[66-68]}$ The dimer is first divided into two fragments corresponding to the monomers; for each fragment a single point calculation is performed obtaining the molecular orbitals (MO); a single point calculation is then performed on the dimer using the fragments' MO as a basis, obtaining the FO couplings $J_{i j}=$ $\left\langle\varphi_{i}|H| \varphi_{j}\right\rangle$ where $\varphi_{i}$ and $\varphi_{j}$ are MO belonging to the two fragments. ${ }^{66,70]}$

The ab initio molecular dynamics (AIMD) simulations are performed with Q-Chem 5.0 at the B3LYP/3-21G* level of theory sampling the canonical ensemble (NVT) using a Langevin thermostat. The time step is set to 0.97 fs. The Fock matrix extrapolation procedure implemented in Q-Chem is used to speed up SCF convergence at each step. 


\section{Acknowledgements}

The authors gratefully acknowledge financial support from Innovation Fund Denmark via the Grand Solutions project "ORBATS" file nr. 7046-00018B.

\section{Keywords: polyanthraquinone $\cdot$ electronic couplings $\cdot$ charge} transport $\bullet$ organic electronics $\bullet$ polymer electrodes

[1] A. Facchetti, Chem. Mater. 2011, 23, 733-758.

[2] M. Kuik, G.-J. A. H. Wetzelaer, H. T. Nicolai, N. I. Craciun, D. M. De Leeuw, P. W. M. Blom, Adv. Mater. 2014, 26, 512-531.

[3] H. Sirringhaus, Adv. Mater. 2014, 26, 1319-1335.

[4] L. Dou, J. You, Z. Hong, Z. Xu, G. Li, R. a. Street, Y. Yang, Adv. Mater. 2013, 25, 6642-6671.

[5] T. M. Swager, Macromolecules 2017, 50, 4867-4886.

[6] J. Yang, Z. Zhao, S. Wang, Y. Guo, Y. Liu, Chem 2018, 4, 2748-2785.

[7] R. Noriega, J. Rivnay, K. Vandewal, F. P. V. Koch, N. Stingelin, P. Smith, M. F. Toney, A. Salleo, Nat. Mater. 2013, 12, 1038-1044.

[8] T. Erdmann, S. Fabiano, B. Milian-Medina, D. A. Hanifi, Z. Chen, M. Berggren, J. Gierschner, A. Salleo, A. Kiriy, B. Voit, et al., Adv. Mater. 2016, 9169-9174.

[9] R. Noriega, Macromol. Rapid Commun. 2018, 1800096

[10] X. Guo, A. Facchetti, T. J. Marks, Chem. Rev. 2014, 114, 8943-9021.

[11] J. L. Brédas, G. B. Street, B. Thémans, J. M. André, J. Chem. Phys. 1985, 83, $1323-1329$

[12] M. M. Toutounji, M. A. Ratner, J. Phys. Chem. A 2000, 104, 8566-8569.

[13] M. B. Goldey, D. Reid, J. de Pablo, G. Galli, Phys. Chem. Chem. Phys. 2016, 18, 31388-31399.

[14] M. Hultell, S. Stafström, Phys. Rev. B 2007, 75, 104304

[15] M. Zhang, H. N. Tsao, W. Pisula, C. Yang, A. K. Mishra, K. Müllen, J. Am. Chem. Soc. 2007, 129, 3472-3473.

[16] S. Wang, M. Kappl, I. Liebewirth, M. Müller, K. Kirchhoff, W. Pisula, K. Müllen, Adv. Mater. 2012, 24, 417-420.

[17] W. Zhang, J. Smith, S. E. Watkins, R. Gysel, M. McGehee, A. Salleo, J. Kirkpatrick, S. Ashraf, T. Anthopoulos, M. Heeney, et al., J. Am. Chem. Soc. 2010, 132, 11437-11439.

[18] D. Venkateshvaran, M. Nikolka, A. Sadhanala, V. Lemaur, M. Zelazny, M. Kepa, M. Hurhangee, A. J. Kronemeijer, V. Pecunia, I. Nasrallah, et al., Nature 2014, 515, 384-388.

[19] Y. Shi, H. Guo, M. Qin, J. Zhao, Y. Wang, H. Wang, Y. Wang, A. Facchetti, X. Lu, X. Guo, Adv. Mater. 2018, 30, 1705745.

[20] D. L. Cheung, D. P. McMahon, A. Troisi, J. Am. Chem. Soc. 2009, 131, 11179 11186 .

[21] A. Troisi, G. Orlandi, Phys. Rev. Lett. 2006, 96, 086601

[22] S. Stafström, Chem. Soc. Rev. 2010, 39, 2484-2499.

[23] D. P. McMahon, A. Troisi, ChemPhysChem 2010, 11, 2067-2074.

[24] N. Vukmirović, L.-W. Wang, J. Phys. Chem. B 2009, 113, 409-15.

[25] D. P. McMahon, D. L. Cheung, L. J. Goris, J. Dacuña, A. Salleo, A. Troisi, J. Phys. Chem. C 2011, 115, 19386-19393.

[26] T. Qin, A. Troisi, J. Am. Chem. Soc. 2013, 135, 11247-11256.

[27] T. Liu, A. Troisi, Adv. Funct. Mater. 2014, 24, 925-933.

[28] A. W. Kohn, D. P. McMahon, S. Wen, T. Van Voorhis, J. Phys. Chem. C 2017, 121, 26629-26636.

[29] H. Bässler, Phys. Status Solidi B 1993, 15, 15-55.

[30] F. Laquai, G. Wegner, H. Bässler, Philos. Transact. A Math. Phys. Eng. Sci. 2007, 365, 1473-87

[31] V. Coropceanu, J. Cornil, D. A. da Silva Filho, Y. Olivier, R. J. Silbey, J. Brédas, Chem. Rev. 2007, 107, 926-952.

[32] N. Vukmirović, L.-W. Wang, Appl. Phys. Lett. 2010, 97, 043305.

[33] A. Troisi, Chem. Soc. Rev. 2011, 40, 2347-58.

[34] R. P. Fornari, A. Troisi, Phys. Chem. Chem. Phys. 2014, 16, 9997-10007.

[35] T. Zhu, T. Van Voorhis, P. de Silva, in Handb. Mater. Model. Methods Theory Model. (Eds.: W. Andreoni, S. Yip), Springer International Publishing, Cham, 2018, pp. 1-31.
[36] D. L. Cheung, A. Troisi, Phys. Chem. Chem. Phys. 2008, 10, 5941-5952.

[37] S. Difley, L.-P. Wang, S. Yeganeh, S. R. Yost, T. V. Voorhis, Acc. Chem. Res. 2010, 43, 995-1004.

[38] N. Vukmirović, Phys. Chem. Chem. Phys. 2013, 15, 3543-3551.

[39] R. P. Fornari, P. W. M. Blom, A. Troisi, Phys. Rev. Lett. 2017, 118, 086601.

[40] L.-W. Wang, N. Vukmirović, Nano Lett. 2009, 9, 3996-4000.

[41] J. Nelson, J. J. Kwiatkowski, J. Kirkpatrick, J. M. Frost, Acc. Chem. Res. 2009, $42,1768$.

[42] A. Troisi, D. L. Cheung, D. Andrienko, Phys. Rev. Lett. 2009, 102, 116602.

[43] L. Meng, Y. Shang, Q. Li, Y. Li, X. Zhan, Z. Shuai, R. G. E. Kimber, A. B. Walker, J. Phys. Chem. B 2010, 114, 36-41.

[44] V. Rühle, A. Lukyanov, F. May, M. Schrader, T. Veho, J. Kirkpatrick, D Andrienko, V. Rühle, A. Lukyanov, F. May, et al., J. Chem. Theory Comput 2011, 7, 3335-3345.

[45] R. Noriega, A. Salleo, A. J. Spakowitz, Proc. Natl. Acad. Sci. U. S. A. 2013, 110 , 16315-16320.

[46] T.-J. Lin, S.-T. Lin, Phys. Chem. Chem. Phys. 2015, 17, 4127-4136.

[47] A. Troisi, A. Shaw, J. Phys. Chem. Lett. 2016, 7, 4689-4694.

[48] H. Etori, T. Kanbara, T. Yamamoto, Chem. Lett. 1994, 23, 461-464.

[49] T. Yamamoto, H. Etori, Macromolecules 1995, 28, 3371-3379.

[50] T. Yamamoto, Y. Muramatsu, B.-L. Lee, H. Kokubo, S. Sasaki, M. Hasegawa, T. Yagi, K. Kubota, Chem. Mater. 2003, 15, 4384-4393.

[51] Z. Song, Y. Qian, M. L. Gordin, D. Tang, T. Xu, M. Otani, H. Zhan, H. Zhou, D. Wang, Angew. Chem. Int. Ed. 2015, 54, 13947-13951.

[52] A. Petronico, K. L. Bassett, B. G. Nicolau, A. A. Gewirth, R. G. Nuzzo, Adv. Energy Mater. 2018, 8, 1700960.

[53] J. Bitenc, K. Pirnat, T. Bančič, M. Gaberšček, B. Genorio, A. Randon-Vitanova, R. Dominko, ChemSusChem 2015, 8, 4128-4132.

[54] B. Pan, J. Huang, Z. Feng, L. Zeng, M. He, L. Zhang, J. T. Vaughey, M. J. Bedzyk, P. Fenter, Z. Zhang, et al., Adv. Energy Mater. 2016, 6, 1600140.

[55] J. Cornil, J.-L. Brédas, J. Zaumseil, H. Sirringhaus, Adv. Mater. 2007, 19, 1791 1799.

[56] G. W. P. van Pruissen, E. A. Pidko, M. M. Wienk, R. A. J. Janssen, J. Mater Chem. C 2014, 2, 731

[57] J. E. Gautrot, P. Hodge, D. Cupertino, M. Helliwell, New J Chem 2006, 30 , 1801-1807.

[58] H. Oberhofer, K. Reuter, J. Blumberger, Chem. Rev. 2017, 117, 10319-10357.

[59] M. B. Goldey, N. P. Brawand, M. Vörös, G. Galli, J. Chem. Theory Comput 2017, 13, 2581-2590

[60] S. M. Gali, G. D’Avino, P. Aurel, G. Han, Y. Yi, T. A. Papadopoulos, V. Coropceanu, J.-L. Brédas, G. Hadziioannou, C. Zannoni, et al., J. Chem. Phys. 2017, 147, 134906

[61] N. E. Jackson, L. X. Chen, M. A. Ratner, Proc. Natl. Acad. Sci. 2016, 113, 8595 8600 .

[62] Y. Liang, Z. Chen, Y. Jing, Y. Rong, A. Facchetti, Y. Yao, J. Am. Chem. Soc 2015, 137, 4956-4959.

[63] Y. Shao, Z. Gan, E. Epifanovsky, A. T. B. Gilbert, M. Wormit, J. Kussmann, A W. Lange, A. Behn, J. Deng, X. Feng, et al., Mol. Phys. 2015, 113, 184-215.

[64] Q. Wu, T. Van Voorhis, J. Chem. Phys. 2006, 125, 164105.

[65] Q. Wu, C.-L. Cheng, T. Van Voorhis, J. Chem. Phys. 2007, 127, 164119.

[66] G. te Velde, F. M. Bickelhaupt, E. J. Baerends, C. Fonseca Guerra, S. J. A. van Gisbergen, J. G. Snijders, T. Ziegler, J. Comput. Chem. 2001, 22, 931-967.

[67] E. J. Baerends, T. Ziegler, A. J. Atkins, J. Autschbach, D. Bashford, O. Baseggio, A. Bérces, F. M. Bickelhaupt, C. Bo, P. M. Boerritger, et al., ADF2017, SCM Theoretical Chemistry, Vrije Universiteit, Amsterdam, The Netherlands, Https://Www.Scm.Com, n.d.

[68] C. Fonseca Guerra, J. G. Snijders, G. te Velde, E. J. Baerends, Theor. Chem Acc. 1998, 99, 391-403.

[69] K. Senthilkumar, F. C. Grozema, F. M. Bickelhaupt, L. D. A. Siebbeles, J. Chem Phys. 2003, 119, 9809-9817.

[70] K. Senthilkumar, F. C. Grozema, C. Fonseca Guerra, F. M. Bickelhaupt, F. D Lewis, Y. A. Berlin, M. A. Ratner, L. D. A. Siebbeles, J. Am. Chem. Soc. 2005 127, 14894-14903. 
Entry for the Table of Contents (Please choose one layout)

Layout 1:

\section{RESEARCH ARTICLE}

Anthraquinone polymers retain conjugation along the backbone and large electronic couplings thanks to interactions between the oxygen's lone pairs and the aromatic system of neighbouring monomers.

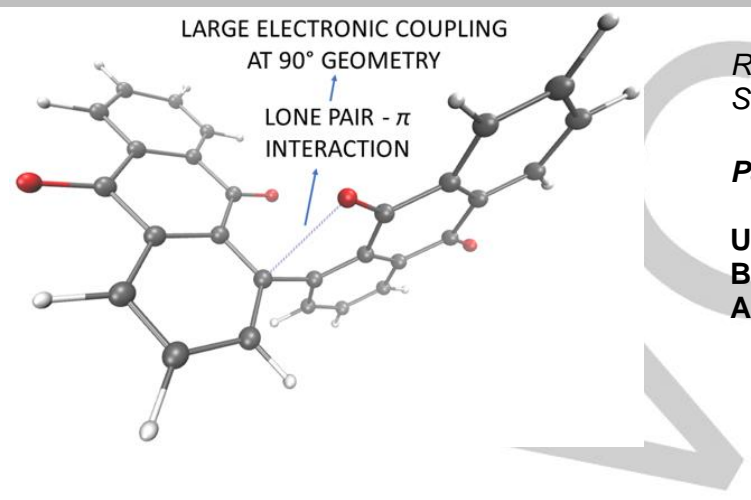

Rocco P. Fornari* and Piotr de Silva*

Page No. - Page No.

Unexpectedly Large Couplings Between Orthogonal Units in Anthraquinone Polymers 